\title{
La flora como recurso terapéutico: el caso de los extractos fluidos de los Laboratorios Codex en México
}

\author{
Paul Hersch Martínez y Miguel García Poujol \\ Instituto Nacional de Antropología e Historia (INAH).osemos@gmail.com
}

Dynamis

[0211-9536] 2008; 28: 329-352
Fecha de recepción: 12 de junio de 2007

Fecha de aceptación: 13 de noviembre de 2007

SUMARIO: 1.-Introducción. 2.-Fuente de referencia: los extractos fluidos de Codex. 3.-La empresa. 4.-Saberes y recursos implícitos en los extractos fluidos de Codex. 5.-Conclusiones.

RESUMEN: Entre las fuentes para una historia social de la terapéutica en México, es pertinente analizar la producción de sus laboratorios farmacéuticos, aún cuando reflejen de manera limitada e indirecta la práctica terapéutica misma. Una primera aproximación a los extractos fluidos de los Laboratorios Codex producidos en el segundo cuarto del siglo XX, permite destacar la diversidad florística de la materia prima empleada en ellos y sus propiedades terapéuticas, avaladas entonces por las autoridades regulatorias. Las indicaciones terapéuticas de los extractos abarcaban una gama amplia de padecimientos, y el 45,8\% de las especies utilizadas eran de procedencia nacional. La figura de los extractos fluidos permitía al clínico acceder al potencial de la flora medicinal, facilitando la flexibilidad prescriptiva en combinaciones y proporciones definidas en función de la condición cambiante del enfermo. Sin embargo, setenta años después, muchos de esos extractos, elaborados por la empresa originada en la empresa Codex, carecen de aval oficial como recursos terapéuticos en sí, incluso en ausencia de estudios que invaliden expresamente las atribuciones registradas entonces. Los productos atestiguan indirectamente, en su origen e indicaciones, un momento de transición en la producción farmacéutica y en la evolución de la terapéutica biomédica en México.

PALABRAS CLAVE: Plantas medicinales, México, regulación sanitaria, medicamentos, investigación clínica.

KEYWORDS: Medicinal Plants, Mexico, sanitary regulation, medications, clinical research. 


\section{Introducción $(*)$}

La terapéutica constituye un área fundamental para la historia social de la medicina, dada su relevancia misma en el ejercicio médico. La terapéutica ha sido determinante en la imagen profesional y la legitimación de los médicos, constituyendo además un indicador útil respecto al papel -real o percibido- del conocimiento científico aplicado en la biomedicina ${ }^{1}$. Sin embargo, la intervención curativa suscita representaciones y prácticas no siempre coherentes entre sí, de modo que el exhorto de Ackernecht ${ }^{2}$ por focalizar en los estudios históricos la conducta de los médicos y no sólo sus ideas o instituciones es particularmente vigente en el campo de la terapéutica, donde a menudo los principios y la teoría han sido asumidos como si fuesen expresiones directas de la práctica misma ${ }^{3}$. Cabe por ello advertir que lo que sigue no se ocupa de la historia de las prácticas terapéuticas en sí, sino de uno de sus referentes indirectos, relativo a una serie de productos de uso medicinal generados en México a partir de diversos vegetales: el catálogo pormenorizado de extractos fluidos distribuido entre médicos y farmacéuticos por los Laboratorios Codex a fines de los años treinta del siglo pasado ${ }^{4}$.

Lejos de suponer una correspondencia llana entre dichos remedios y el ejercicio terapéutico de los clínicos en esa época, pretendemos explorar algunas de sus implicaciones significativas: a) el origen de los saberes y recursos inherentes a esos extractos, b) el tipo de práctica prescriptiva a que correspondían indirectamente y c) su carácter como marcadores de un momento determinado del proceso de validación de recursos terapéuticos en México.

Este trabajo se centra en un momento crítico del proceso de transición terapéutica en la biomedicina mexicana, pues es en el segundo cuarto del

$\left(^{*}\right)$ Agradecemos a Jorge Ebrard (Laboratorios Mixim) y Rolf Stein (Central de Drogas) por sus comentarios e información, a los evaluadores anónimos de este trabajo por sus observaciones, y al Consejo Nacional de Ciencia y Tecnología (México) por su apoyo a través del Proyecto Sectorial Salud (clave 2003-C01-18/A-1).

1. Warner, John Harley. The therapeutic perspective. Medical practice, knowledge and identity in America, 1820-1995. Princeton: Princeton University Press; 1997.

2. Ackerknecht, Erwin. A Plea for a «behaviorist» approach in writing the History of Medicine. Bulletin of the History of Medicine. 1967; 22: 211-214.

3. Warner, n. 1, p. 3.

4. Carlos Stein y Cía. Descripción Técnica de los Extractos Fluidos Medicinales. México, s/f. 
siglo XX que se llevan a cabo dos reformas regulatorias significativas, relativas al registro y autorización oficial de medicamentos en el país. La primera reforma tuvo lugar en 1927 y $1928^{5}$ y la segunda en $1942^{6}$, coincidiendo en esa época con el momento de fundación del Instituto Mexicano del Seguro Social (1944) y con el arranque del proceso de consolidación industrial del país; es además en los años que van de 1925 a 1950 cuando se establecen y consolidan en México las principales empresas farmacéuticas extranjeras que dominarían el mercado ${ }^{7}$, cuando alcanzan su culmen algunas firmas nacionales en ese ramo que luego perderían su impacto innovador, como fue el caso de los mismos Laboratorios Codex y del Laboratorio Químico Central, fabricante de la línea de medicamentos $\mathrm{Garcol}^{8}$; asimismo, en ese periodo se modifica radicalmente el perfil de la Farmacopea Nacional como referente oficial ${ }^{9}$ y se consolida un modelo más riguroso de autorización y registro sanitario de medicamentos ${ }^{10}$.

Si bien en este trabajo no se pretende determinar en qué medida incidieron en la práctica médica todos estos procesos de orden farmacéutico, industrial y regulatorio, ellos constituyeron factores contextuales determinantes en la transición progresiva de la terapéutica biomédica en México. De manera refleja a lo que sucedía en todo el mundo occidental, el periodo a que nos referimos comprende el surgimiento de las sulfamidas y los primeros antibióticos ${ }^{11}$, así como en 1944 la fundación de la empresa

5. Departamento de Salubridad Pública. Reglamento para el registro y certificado de medicinas de patente, especialidades y productos de tocador, higiénicos, de belleza y demás similares. Diario Oficial de la Federación. México, 6 de septiembre de 1928, p. 3-6 (promulgado el 17 de agosto de 1928); Bermúdez, Salvador. Las medicinas «de patente» y la salubridad pública. Gaceta Médica de México. 1929; 60: 208-219.

6. Departamento de Salubridad Pública. Reglamento para el registro, revisión, certificación y propaganda de medicinas de patente, especialidades, aparatos médicos, productos de tocador, higiénicos, de belleza y similares. Diario Oficial de la Federación. México, 20 de Mayo de 1942: 15-20 (promulgado el 8 de mayo de 1942).

7. Gomezjara, Francisco. Fábricas y supermercados de salud. In: Mercado, Francisco, ed. Medicina ¿para quién? México: Unión Nacional de Médicos/Nueva Sociología; 1980, p. 167-312.

8. Hersch Martínez, Paul. La industrialización químico-farmacéutica mexicana y la flora: el caso de los Laboratorios Garcol. Boletín de la Sociedad Química de México. 2008; 1 (1): 5-12.

9. Hersch Martínez, Paul. La Farmacopea Nacional y el estatuto terapéutico de la flora en la biomedicina mexicana. Dynamis. 2001; 21: 375-407.

10. Hersch Martínez, Paul. Plantas medicinales: relato de una posibilidad confiscada. El estatuto de la flora en la biomedicina mexicana. México: Instituto Nacional de Antropología e Historia; 2000, p. 421-427. Departamento de Salubridad Pública, n. 5 y 6.

11. Fue en 1944 cuando se introdujo oficialmente la penicilina en México, en un decreto cuya redacción misma refleja el impacto que habían generado sus aplicaciones iniciales: Secre- 
Syntex en México, donde se obtendrían por primera vez a escala industrial diversos esteroides, partiendo de la planta denominada barbasco (Dioscorea composita); es a su vez en esa época que en toda América Latina se inicia el abandono progresivo de la terapéutica como asignatura en los planes universitarios de estudio en medicina y su sustitución por la materia de farmacología ${ }^{12}$; son los años que preceden inmediatamente a las décadas en que se presentaron las mayores tasas anuales de incorporación de fármacos de síntesis al mercado a nivel mundial, en un incremento sostenido que tuvo su culmen en 1975, para desde entonces descender progresivamente ${ }^{13}$.

Es en ese periodo determinante, previo a la época de mayor expansión y consolidación de la farmacoterapia moderna en la biomedicina, que se afina y afianza la investigación experimental centrada en principios moleculares activos ${ }^{14}$.

La práctica de la terapéutica había iniciado su lenta transformación a partir del nacimiento de la farmacología experimental, con desarrollos pioneros en los siglos XVII y XVIII ${ }^{15}$; esa transformación implicó el paso de un modelo terapéutico que se derivaba principalmente de la interacción del médico con sus pacientes y colegas, a uno - vigente hoy- de mayor sumisión a la ciencia proveniente del laboratorio experimental ${ }^{16}$. En palabras del clínico mexicano Mario Salazar Mallén, al rememorar en 1960 los cambios vividos en su propio ejercicio médico iniciado en 1929, no sólo los antibióticos y los agentes quimioterápicos antibacterianos transformaron la fisonomía clínica de algunas infecciones y anularon el interés clínico de otras, sino que los adelantos de la industria química y farmacéutica influyeron decisivamente en el arte de la prescripción, haciendo desaparecer

taría de Salubridad y Asistencia. Decreto que declara de Interés Público para la salubridad general la fabricación, elaboración, comercio, importación, transporte, suministro y uso de la Penicilina. Diario Oficial de la Federación. México, 20 Marzo 1944 (promulgado el 18 de febrero de 1944).

12. Hersch Martínez, n. 10, p. 186-203.

13. Pignarre, Philippe. El gran secreto de la industria farmacéutica. Barcelona: Gedisa; 2005, p. 48.

14. Marks, Harry. The progress of experiment, science and therapeutic reform in the United States, 1900-1990. Cambridge: Cambridge University Press; 1997. Pignarre, n. 13, p. 95-105.

15. Maehle, Andreas-Holger. Drugs on trial: Experimental pharmacology and therapeutic Innovation in the eighteenth century. Amsterdam: The Wellcome Trust; 1999 [Col. Clio Medica na. 53].

16. Warner, n. 1, p. 7. 
prácticamente la denominada «prescripción magistral» de casi todas las ramas de la medicina a excepción de la dermatología ${ }^{17}$.

En este contexto, la figura de los extractos fluidos de origen vegetal remite a un modelo de medicamento y de prescripción enmarcado en una terapéutica que iba cayendo en desuso, donde la pretendida atención a la especificidad del enfermo sería desplazada por el énfasis en la especificidad de la enfermedad y en la estandarización de procedimientos y recursos ${ }^{18}$.

\section{Fuente de referencia: los extractos fluidos de Codex}

Ahora bien, en relación con la obra de referencia que nos ocupa, se ha destacado ya el carácter heterogéneo y la trascendencia diferencial de las fuentes de información de utilidad para la historia social de la terapéuti$\mathrm{ca}^{19}$. Entre los textos de enseñanza y los diarios de médicos practicantes y a su vez, entre la correspondencia de médicos con familiares de la misma profesión y los discursos universitarios y académicos, se encuentra un tipo de material muy condicionado y en absoluto exento de sesgos: el producido por empresas fabricantes y comercializadoras de remedios y medicamentos. Esta gama de materiales, variada en sí, coincide sin embargo en el cometido común de anunciar mercancías, con toda la carga de distorsión publicitaria que ello acarrea, que puede ser mayor o menor en función de la calidad o la seriedad de las empresas interesadas en la realización comercial de sus productos.

La figura de la obra que presenta recursos terapéuticos motivados por intereses comerciales no es reciente en México. Incluso el Libellus de Medicinalibus Indorum Herbis, la célebre obra del médico indígena Martín de la Cruz, ilustrada por Juan Badiano, tenía entre sus propósitos el poco conocido de presentar en Europa una relación de plantas de uso curativo mercantilmente prometedoras ${ }^{20}$; otra obra de connotaciones similares es la

17. Salazar Mallén, Mario. La evolución del ejercicio de la medicina en los últimos cincuenta años. Salud Pública de México. 1960; 2 (1): 161-166.

18. Warner, n. 1, p. 58-80.

19. Warner, n. 1, p. 345-351.

20. De la Cruz, Martín. Libellus de Medicinalibus Indorum Herbis [1552]. México: Instituto Mexicano del Seguro Social; 1991. La obra fue elaborada por encargo de Francisco de Mendoza, hijo del virrey Antonio de Mendoza, cuya familia exportaba plantas medicinales a Europa. Véase Viesca Treviño, Carlos. El primer libro médico mexicano. Ciencias. 1991; 21: 37-40. 
escrita por el médico sevillano Nicolás Monardes introduciendo en Europa plantas de la Nueva España reputadas como medicinales ${ }^{21}$. Los catálogos comerciales propiamente dichos, de aparición posterior, constituyen referentes directos e indirectos de la práctica terapéutica, incluyendo la que se realiza en el ámbito doméstico ${ }^{22}$. Esta gama abarca además publicaciones que agrupan la producción de diversos laboratorios y que desplazaron progresivamente a las farmacopeas como textos de referencia prescriptiva $^{23}$; en el caso de México corresponden al Diccionario de Especialidades Farmacéuticas que se publica desde 1944, teniendo entre sus antecedentes en el caso de Francia al Diccionario Vidal cuyo arranque data de $1913^{24}$.

En ese contexto, la fuente de referencia a que aludimos centralmente es la publicación denominada Descripción Técnica de los Extractos Fluidos Medicinales Codex, aparecida sin fecha precisa, posiblemente en la segunda mitad de la década de los años treinta y/o entre 1942 y $1944^{25}$. En ese material de promoción dirigido explícitamente «a los Honorables Cuerpo Médico y Farmacéutico de la República Mexicana y de Centro y Sur América», la empresa productora daba a conocer que la investigación requerida para conformar las descripciones e indicaciones de los extractos y de las especies de origen contenidos en la obra, había contado con la participación de tres connotados profesores: el biólogo Isaac Ochoterena, el botánico Maximino Martínez y el farmacéutico Amador E. Ugalde, además del químico-farmacéutico Manuel David Garza, de los mismos Laboratorios Codex ${ }^{26}$. Ochoterena, Martínez y Ugalde fueron figuras muy relevantes en el ámbito académico de entonces. Isaac Ochoterena (1885-1950) jugó un

21. Monardes, Nicolás, Primera y segunda y tercera partes de la Historia medicinal de las cosas que se traen de nuestras Indias Occidentales [Sevilla, 1574], México: Instituto Mexicano del Seguro Social; 1990.

22. Hersch Martínez, Paul. Tres textos de medicina doméstica en México: Velasco, Barajas y López Tilghman. Boletín Mexicano de Historia y Filosofía de la Medicina. 2004; 7 (1): 11-18.

23. Hersch Martínez, n. 10, p. 224-225, 239, 403.

24. Landero, et al., eds. Diccionario de especialidades farmacéuticas. México: P.L.M.-Agencia de propaganda para los médicos, S. de R. L.; 1944. Dictionnaire Vidal. París: Editions du Vidal; 1991.

25. Stein y Cía, n. 4. Cabe mencionar como rasgo indicativo de su fecha de aparición o reimpresión la nota en su portada, referida a la empresa: «Administrada por la H. Junta de Vigilancia de la Propiedad Extranjera», la cual se formó para administrar las empresas confiscadas durante la Segunda Guerra Mundial, como veremos más adelante. Stein, Rolf. México, 2007 (comunicación personal).

26. Stein y Cía, n. 4, p. 5. 
papel fundamental en el proceso de institucionalización de la biología en México y ejercía en esa época el cargo de director del Instituto de Biología de la Universidad Nacional Autónoma de México (UNAM) del que fue fundador y dirigió durante 17 años ${ }^{27}$.

A su vez, Maximino Martínez (1888-1964) fue uno de los referentes de la moderna botánica en México; dirigió el Departamento de Botánica de la Dirección de Estudios Biológicos, que luego se convertiría en el referido Instituto de Biología de la UNAM. Introdujo el sistema de Adolfo Engler para catalogar y clasificar a las plantas mexicanas, aplicado en el Herbario Nacional y en otros herbarios del país. Fundó en 1941 la Sociedad Botánica de México y en 1944 ingresó como investigador al referido Instituto de Biología, donde trabajó hasta su muerte. Martínez publicó numerosas obras de relevancia sobre taxonomía y botánica económica, destacando entre ellas su Catálogo alfabético de nombres vulgares y científicos de plantas que existen en México publicado entre 1923 y 1929, con una segunda edición en 1937; Las plantas mas útiles que existen en la República Mexicana con tres ediciones sucesivas entre 1928 y 1959; Las Plantas Medicinales de México, con cuatro ediciones sucesivas entre 1933 y 1959; y finalmente la moderna versión del Catálogo de Nombres Vulgares y Científicos de Plantas Mexicanas editado por el Fondo de Cultura Económica en forma póstuma ${ }^{28}$.

Amador Ugalde, reconocido farmacéutico, propietario de la «Botica del Hospital Real» en la ciudad de México, anunciada como «antigua botica alemana», presidió en 1913 la «Liga de Farmacéuticos Mexicanos» ${ }^{29}$ y fue en noviembre de 1916 uno de los fundadores de la Sociedad Farmacéutica Mexicana ${ }^{30}$; años después, encabezándola, pugnó sistemáticamente por el reconocimiento profesional y la regularización industrial de la farmacia,

27. Ledesma Mateos, Ismael; Barahona Echeverría, Ana. Alfonso Luis Herrera e Isaac Ochoterena: la institucionalización de la biología en México. Historia Mexicana. 1999; 48 (3): 635-673; Herrera, Teófilo et al. Breve Historia de la Botánica en México. México: Fondo de Cultura Económica; 1998.

28. Valdez, Javier. Maximino Martínez, 1888-1964. In: Grupo Etnobotánico Latinoamericano, http://www.ibiologia.unam.mx/gela/p-3.html; Herrera et al., n. 27, p. 111.

29. El Diario. México. 6 Sept. 1913: 4.

30. Entre otros socios fundadores figuraban los connotados farmacéuticos Juan Manuel Noriega, Miguel Cordero, Ignacio López Valdez y Triunfo Bezanilla. Nota informativa en: La Farmacia. México. 1 Ago. 1917: 39-40. 
así como por el impulso a la farmacopea y también por el cultivo y el cabal aprovechamiento de la flora medicinal en el país ${ }^{31}$.

Hay que subrayar sin embargo algo significativo, y es que en esta relación de asesores de la empresa se encuentra ausente la figura de los médicos: a la perspectiva botánica, farmacéutica y química no se incorporaba, ya desde entonces, la proveniente de la práctica clínica en sí. La paradoja de que en la lectura de las propiedades terapéuticas de la flora no figure ni destaque el clínico, expresa indirectamente la exclusión de la flora como recurso terapéutico en los diversos escenarios de la biomedicina actual.

Los extractos fluidos de Codex se vendían en diversas presentaciones y su etiqueta descriptiva consignaba datos farmacológicos, terapéuticos y de posología, al estar orientados al uso médico y farmacéutico, lo cual es relevante porque implicaba el acceso del público usuario a dicha información. En el catálogo de Codex, la información relativa a cada extracto incluía también una descripción botánica de cada especie o en su caso, la descripción farmacognósica de la droga en cuestión, así como una ilustración de cada planta y la composición química del extracto, además de precisar las dosis correspondientes para su aplicación en pacientes, su número de registro ante la Secretaría de Salubridad y Asistencia y algunas notas precautorias ${ }^{32}$.

La figura de los extractos fluidos había sido introducida en el mercado farmacéutico en los Estados Unidos por la empresa Parke Davis and Company en 1879, al presentar la preparación fluida de cornezuelo de centeno, denominada Liquor Ergotae Purificatus, bajo el principio de que cada lote fuese de carácter uniforme. Para 1883, la empresa anunció sus veinte primeras «preparaciones fluidas uniformes» o extractos fluidos normalizados mediante alguna forma de ensayo ${ }^{33}$. Dicha modalidad extractiva aportaba la ventaja de una dosificación más exacta, al equivaler cada mililitro de extracto fluido a un gramo de la droga seca. Ello implicó un avance de consideración en el manejo de los extractos vegetales de uso terapéutico; un mayor grado de estandarización de la materia médica de origen vegetal permitiría entonces la prescripción combinada de diversas especies por el

31. Hersch Martínez, n. 10, p. 288, 324, 384-385. Ugalde, Amador. En el congreso de droguistas y propietarios de boticas. La Farmacia. 1927; 2 (25): 385-388.

32. Stein y Cía., n. 4.

33. Manual de Terapéutica. Breve descripción de algunas de las preparaciones biológicas y farmacéuticas manufacturadas por Parke Davis \& Co. Nueva York: Parke Davis and Co.; 1927. 
médico y el despacho de sus recetas en las farmacias. Por añadidura, a partir de un extracto fluido como base, surgió la posibilidad de preparar con mayor facilidad jarabes, vinos y tinturas de uso medicinal. De hecho, al término de los años treinta, Codex no era la única firma que en México tenía registrados como medicamentos extractos fluidos de plantas ante el Departamento de Salubridad Pública: empresas como Eli Lilly and Co., Sharp \& Dhome y J. Busch obtuvieron registros - que luego perdieron - en la misma época que los Laboratorios Codex, como más adelante veremos. Codex también había registrado e introducido al mercado mexicano productos intermedios para los farmacéuticos, con la idea de facilitar la preparación de remedios tradicionales de botica: se trataba de extractos para elaborar, entre otros, Bálsamo de Opodeldoc, Aguardiente Alemán, Bálsamo Tranquilo, Jarabe de Desessartz y Vino Yodotánico ${ }^{34}$.

\section{La empresa}

Los Laboratorios Codex, fundados en México por industriales franceses a inicios del siglo XX, fueron luego adquiridos al principiar la década de los años veinte por el empresario alemán Carlos Stein, propietario a su vez de la casa farmacéutica Carlos Stein y de la «Droguería Cosmopolita» de la ciudad de México ${ }^{35}$. En 1942, al involucrarse el país como beligerante en la Segunda Guerra Mundial al lado de los Aliados, las empresas de propiedad alemana, japonesa e italiana fueron confiscadas por las autoridades gubernamentales, con lo cual la firma Codex pasó a ser administrada entonces por el Estado mexicano mediante una Junta de administración y vigilancia de la propiedad extranjera cuyas funciones continuarían hasta $1950^{36}$. Aún en esas circunstancias, sus extractos figuraron en el recién creado Diccionario de Especialidades Farmacéuticas, pero solamente en su segunda edición, aparecida en $1945^{37}$.

\footnotetext{
34. Stein y Cía., n. 4, p. 178-181.

35. Stein, Rolf. México, 2007 (comunicación personal).

36. Archivo General de la Nación (México) (en adelante, AGN), RP/MAV, 705.1/108, citado en: Niblo, Stephen R. Allied policy toward axis Interests in Mexico during World War II. Mexican Studies/Estudios Mexicanos. 2001; 17 (2): 351-373; Hersch Martínez, 2000, n. 9, p. 354-367.

37. Landero, Ignacio; Ferester, Marc; Kestemberg, Juan; Rosenstein, Emilio, eds. Diccionario de Especialidades Farmacéuticas. México: P.L.M. Agencia de propaganda para los médicos; 1945.
} 
En unos cuantos años, la empresa habría de enfrentarse no sólo a la intervención gubernamental a causa de la Segunda Guerra Mundial ${ }^{38}$, sino a la cancelación del registro de muchos de sus productos en 1944 y 1948, destacando entre ellos su línea de extractos fluidos, como efecto del decreto regulatorio de $1942^{39}$. Amparados en la regulación de 1928, dichos extractos habían sido registrados como medicamentos por la Dirección de Salubridad Pública en 1935 y $1936^{40}$. Para completar el cuadro de complicaciones de la empresa, en 1947 se generó un movimiento huelguístico entre los trabajadores, sin aparente conexión con ello, se desencadenó un incendio que consumió la totalidad de las bodegas ${ }^{41}$.

Tras la Segunda Guerra Mundial, el gobierno mexicano expidió en diciembre de 1949 un decreto presidencial que anunciaba la formación, a partir de esas compañías confiscadas, de una empresa nacional productora de medicamentos de utilidad pública ${ }^{42}$; dicho proyecto daría lugar en 1953 a la formación de la Industria Nacional Químico Farmacéutica, cuyo director fue el reconocido médico Salvador Zubirán; en ella se incluyeron las empresas confiscadas Bayer, Behring, Beick-Félix, Carlos Stein - de quien dependían los Laboratorios Codex-, Fábrica de ácidos La Viga, Knoll, Merck y Schering ${ }^{43}$. Sin embargo, dicha iniciativa no prosperó, reintegrándose las empresas en los años subsiguientes a sus propietarios originales, aunque en el caso de los Laboratorios Codex, ya desde finales de 1948 el mismo grupo de empresarios fundadores le dieron continuidad estableciendo una nueva

38. Departamento de Salubridad Pública, n. 6.

39. Secretaría de Salubridad y Asistencia. Lista de los registros de productos medicinales, que se cancelan por no haber cumplido los interesados con los requisitos que exigen el Reglamento y demás leyes relativas. Diario Oficial de la Federación. 7 Sept. y 28 Dic. 1944. Véase por ejemplo: Secretaría de Salubridad y Asistencia. Lista de los registros de productos medicinales, que se cancelan por no haber cumplido los interesados con los requisitos que exigen el Reglamento y demás leyes relativas. Diario Oficial de la Federación. México. 31 Ago. 1946, 14 Feb. 1947, 20 Ago. y 7 Sept. 1948.

40. Véase por ejemplo: Departamento de Salubridad Pública. Lista número 18 de los productos medicinales que han sido aprobados. Diario Oficial de la Federación. México. 8 Feb. 1935; Lista número 19 de los productos medicinales que han sido aprobados. Ibidem. 5 Mar. 1936. Lista número 20 de los productos medicinales que han sido aprobados. Ibidem. 1 Ago. 1936.

41. El Universal, 24 y 26 Jun. 1947: 12. 300 mil pesos perdidos en el incendio de unos laboratorios. El Universal, 13 Jul. 1947: 1 y 15.

42. Secretaría de Salubridad y Asistencia. Decreto por el cual se crea un Laboratorio Nacional de Medicamentos de Participación Estatal. Diario Oficial de la Federación. México. 2 Dic. 1949.

43. Hersch Martínez, n. 10, p. 354-367; Gómezjara, n. 7, p. 235 y ss. 
firma, denominada «Laboratorios Mixim» ${ }^{44}$. Al cancelarse sus registros, los extractos fluidos de Codex fueron retirados de la dispensación directa y de la promoción como sustancias de uso medicamentoso ${ }^{45}$; al cambiar de estatuto, recibieron licencia exclusivamente como materia prima para la industria farmacéutica, lo que constituye su condición regulatoria actual; a su vez, parte de la producción del antiguo laboratorio Codex continuó a cargo de la casa denominada Laboratorios Wiener Codex, ubicada en el estado de San Luis Potosí, en México ${ }^{46}$.

En el transcurso de los años cincuenta, los Laboratorios Mixim dieron continuidad a la producción de extractos fluidos que fabricaba Codex, al tiempo que los productos «Codex-Stein», bajo jurisdicción de la empresa Wiener, abandonaron el campo de los extractos naturales para adoptar un perfil acorde al momento de consolidación de la farmacoterapia de síntesis. Para 1960, los «Productos Codex-Stein» aparecían enlistados bajo el registro general de la empresa Wiener en la octava edición del Diccionario de Especialidades Farmacéuticas y en ellos había desaparecido el antiguo perfil de los extractos vegetales que Codex anunciaba quince años antes, en la segunda edición de esa obra. Para entonces, ya una parte significativa de los productos Codex incluían en su composición principios químicos definidos o sintéticos (adrenalina, fenobarbital, sulfatiazol, diyodo-oxiquinoleina, sulfas, emetina, codeina, bromuro de estroncio, etc) ${ }^{47}$.

En 1961, los Laboratorios Mixim fueron adquiridos por la empresa norteamericana S.B. Penick, teniendo entre sus propósitos principales iniciar la producción de papaína en México. Para 1977, en un momento en que el giro original de la empresa basado en productos naturales no resultaba ya relevante para sus propietarios, y como consecuencia de la fusión de la empresa Penick con otra compañía, Mixim fue vendida a los

44. Véase: http://www.labmixim.com/Espanol/Historia.html.

45. Desde 1949, cancelados los registros de sus extractos fluidos, los 91 productos que Codex consignaba en el Diccionario de Especialidades Farmacéuticas contenían ya muy pocos vegetales, muchos de ellos jarabes, vacunas y serovacunas que luego serían retirados del mercado. Para 1954, la empresa ya no consigna productos en la publicación. Landero, Ignacio; Rosenstein, Emilio, eds. Diccionario de Especialidades Farmacéuticas. México: P.L.M. Agencia de propaganda para los médicos; 1949, p. 656-657. Landero, Ignacio; Rosenstein, Emilio, eds. Diccionario de Especialidades Farmacéuticas. México: P.L.M. Agencia de propaganda para los médicos; 1954.

46. Ebrard, Jorge. Laboratorios Mixim. México, 2007 (comunicación personal).

47. Landero, Ignacio; Rosenstein, Emilio, eds. Diccionario de Especialidades Farmacéuticas. México: P.L.M. Agencia de propaganda para los médicos; 1960. 
actuales propietarios mexicanos que ya trabajaban en ella desde $1962^{48}$, diversificando progresivamente su producción de extractos naturales para abastecer, además de la farmacéutica, a otros ramos de la industria, como el alimentario, el de condimento, el de la perfumería y el de cosmética.

\section{Saberes y recursos implícitos en los extractos fluidos de Codex}

Varios son los rasgos destacables que resultan de un análisis general de los extractos fluidos de Codex publicados en su catálogo. Por problemas de espacio hemos decidido publicar los diferentes cuadros de los extractos fluidos en la página web abajo indicada. Hemos incluido, además, su nomenclatura y su origen vegetal, así como sus indicaciones y contraindicaciones ${ }^{49}$. Aparte de los preparados de composición múltiple, se trata de 162 extractos fluidos involucrando a 155 especies, dado que algunas de éstas eran origen de diversos extractos dependiendo de la parte utilizada o su preparación, como son los casos del benjui, castaño de indias, hamamelis, lechuga, naranjo, quina y tolú ${ }^{50}$. Como se manifiesta en sus mismos nombres, las plantas utilizadas como materia prima presentaban diversa procedencia, destacando términos de origen indígena (estafiate, chiltipiquín, güiro, chachaca, cuachichile, toloache, papaconi, tomahuactlacopatli, tatlancuaya, huachácata, tilxóchitl, tlachichinole, anacahuite, quahayohuachtli, chichiquahuitl, tlacochichic, cuanaxonaxi, zacatechichi, etc.) pero también términos foráneos, propios de la farmacognosia cosmopolita de entonces (bucco de Africa, cardamomo menor, cato, columba, cáprago, visco, cubeba, jaborandi, etc.). El análisis de las propiedades medicinales de los extractos tal como se publicitan, refleja a su vez indicaciones correspondientes a enfermedades que siguen siendo reconocidas como tales en la biomedicina (arterioesclerosis, asma, pielonefritis, varices, etc.), pero también incluye entidades nosológicas raramente referidas en la actualidad, como los ubicuos «catarros no respiratorios» (catarro de vías urinarias, de vías biliares, de la vejiga) o la «atonía gástrica» ${ }^{51}$.

\footnotetext{
48. Ebrard, n. 46.

49. La investigación antropológica en el INAH, Proyectos colectivos: Actores sociales de la flora medicinal en México: V. Véase http://www.antropologia.inah.gob.mx/htm/f_presentacion.htm

50. Véase n. 49.

51. Cabe recordar que el término genérico «catarro» proviene de katarrhein: «fluir hacia abajo». Véase: Gómez de la Serna, Guido. Breve Diccionario Etimológico de la Lengua Española. México: Fondo de Cultura Económica; 1988, p. 155.
} 
Un rasgo a destacar en las indicaciones de los productos Codex, común en la época, es la inclusión regular de términos clásicos que luego cayeron progresivamente en desuso, utilizados para describir el efecto de los extractos «simples»: carminativo, béquico, tónico, emoliente, astringente, pectoral, estomáquico, anhidrótico, balsámico, tonicardiaco, rubefaciente, galactógeno, eupéptico, antiperiódico, sialagogo, drítico, alterante, vulnerario, amargo, emenagogo, revulsivo, anexosmótico, eupnéico; algunos de éstos vocablos y otros más del mismo corte refieren a una terminología que hoy puede parecer peculiar pero que se enmarca también en la perspectiva del farmacéutico y no sólo del clínico. En algunos casos, esos términos fueron progresivamente desplazados, pero no porque las especies o extractos careciesen de dichos efectos, o por desaparecer del horizonte sintomatológico o epidemiológico actual la afección que entonces combatían como, por ejemplo, la cantidad insuficiente de saliva que motivaría el uso de un remedio sialagogo, la carencia de leche en la madre que requeriría un galactógeno o el exceso de sudor que exigiría un anhidrótico. Más bien expresan una determinada manera de concebir y construir la terapéutica, donde el clínico contaba aún con cierto margen de autonomía y creatividad en el proceso prescriptivo, individualizando su tratamiento en función de la relevancia diferencial de los signos y síntomas de un determinado paciente, pues como bien se sabe, lejos de ser uniformes, las manifestaciones individuales de una determinada enfermedad varían dependiendo de la condición particular de los enfermos.

Las expresiones de los efectos de esos remedios se han de ligar con las necesidades de tratamiento sintomático y de base. Esas expresiones subrayan el efecto tangible del remedio y pertenecen a un «universo operativo» clínico, de índole general. Si los contrastamos con términos más recientes, ya permeados por una mayor precisión etiológica o fisiopatológica, vemos que la caracterización misma de los efectos ha evolucionado de manera refleja y significativa para ubicarse ahora a un nivel molecular. Así, la adjudicación de un efecto terapéutico conlleva un contexto determinado: por ejemplo, cuando se habla de un extracto «vulnerario»o «emoliente», nos ubicamos en un nivel aún general pero con un margen intermedio de precisión, que no atiende al orden etiológico, sino sintomático, privilegiando la manifestación corroborable en la clínica. En cambio, cuando hablamos hoy de un «bloqueador de los canales de calcio», de un «agente inmunosupresor», de un «antagonista muscarínico colinérgico», se atiende al modo de acción del fármaco en la respuesta biológica que genera, en lo que se ha referido de 
la terapéutica médica actual como un «torneo molecular» ${ }^{52}$. El producto medicamentoso conlleva un universo de referencia que se plasma en los efectos que se le adjudican.

Ahora bien, en el conjunto de las propiedades medicinales atribuidas a los extractos que nos ocupan, destacan las indicaciones relativas a afecciones respiratorias y digestivas, seguidas por las febriles de diverso origen, las urinarias, las sedantes nerviosas, las gineco-obstétricas y las dermatológicas. Sin embargo, la gama también abarca, entre otros, problemas reumatológicos y cardiovasculares en ese orden de importancia. También es notoria en el listado de extractos fluidos la existencia de especies de efecto estupefaciente o psicoactivo, respecto a los cuales la empresa consignaba claras notas admonitorias, sin por ello soslayar el margen de utilidad terapéutica que entonces se les reconocía. Los extractos fluidos de café y de tabaco presentan un perfil semejante, al ser reconocido su efecto medicinal que rebasa el uso popular que los caracteriza, el primero como «excitante de la circulación, antisomnífero y diurético por acción refleja», y como «antídoto de algunos narcóticos»; el segundo como «antihelmíntico en enemas, antidoloroso e insecticida». A su vez en relación a las partes de las especies utilizadas como materia prima, destacan las raíces y rizomas $(28.2 \%)$, las hojas $(20.8 \%)$ y las cortezas $(13.4 \%)$.

La distancia entre la lectura popular o empírica del efecto y su corroboración experimental se expresa también en esa relación de extractos; en lo que refiere a sus indicaciones, al margen de que exigen un detenido estudio que las confronte con la información experimental disponible hoy, en diversos casos esas atribuciones de entonces se encuentran en la actualidad sustentadas en la literatura, como sucede con especies tan conocidas como el tomillo (Thymus vulgaris) en su efecto antiséptico de las vías respiratorias ${ }^{53}$, la uva ursi (Arctostaphylos uva ursi) indicada en las «afecciones catarrales de la vejiga», dado su documentado efecto antiséptico urinario ${ }^{54}$, el tilo (Tilia europaea) en su efecto sedante ${ }^{55}$, el eucalipto

\footnotetext{
52. Dossey, Larry. Tiempo, espacio y medicina. Barcelona: Ed. Kairós; 1986.

53. Basch, E. et al. Thyme (Thymus vulgaris L.), thymol. Journal of Herbal Pharmacotherapy. 2004; 4 (1): 49-67.

54. Yarnell, E. Botanical medicines for the urinary tract. World Journal of Urology. 2002; 20 (5): 285-293.

55. Coleta, M. et al. Comparative evaluation of Melissa officinalis L., Tilia europaea L., Passiflora edulis Sims. and Hypericum perforatum L. in the elevated plus maze anxiety test. Pharmacopsychiatry. 2001; 34 (Suppl. 1): S20-521.
} 
(Eucalyptus globulus) en su acción antiséptica en vías respiratorias ${ }^{56}$, el chaparro amargoso (Castela texana) como amebicida ${ }^{57}$ o la comelina (Commelina pallida) como hemostática ${ }^{58}$. Efectos no consignados entonces de plantas incluidas en la producción de Codex han sido luego documentados experimentalmente, como es el caso de la acción sedante de la valeriana mexicana (Valeriana procera) ${ }^{59}$ o el efecto hipotensor del zapote blanco (Casimiroa edulis $)^{60}$, mientras que no figuran en la relación de extractos de Codex especies mexicanas de uso popular frecuente entonces y ahora, y cuyo efecto terapéutico se encuentra hoy precisado experimentalmente, como es el caso del cuachalalate (Amphipterygium adstringens) ${ }^{61} \mathrm{o}$ el árnica mexicana (Heterotheca inuloides) ${ }^{62}$.

El cuadro 2 muestra el origen del abasto de la materia prima vegetal empleada ${ }^{63}$. Se pueden agrupar las especies en términos generales en: a) las procedentes del extranjero; b) las que siendo de origen foráneo eran ya producidas o colectadas en México y c) las propiamente originarias del país y en su gran mayoría silvestres. Estos dos últimos grupos en conjunto suman 71 , lo que equivale al $45,8 \%$ del total de especies ofrecidas en forma de extractos fluidos por Codex. Tal como se consigna en la columna derecha de dicho cuadro, la gran mayoría de los extractos contaban con registro expedido por el Departamento de Salubridad Pública; además, al estar dirigido al cuerpo médico y farmacéutico, el catálogo consignaba dosis

56. Salari, M.H. et al. Antibacterial effects of Eucalyptus globulus leaf extract on pathogenic bacteria isolated from specimens of patients with respiratory tract disorders. Clinical Microbiology and Infection. 2006; 12 (2):194-196.

57. Reyes-López, M. et al. The amoebicidal aqueous extract from Castela texana possesses antigenotoxic and antimutagenic properties. Toxicology in vitro. 2005; 19 (1): 91-97.

58. Pérez-Cirera, M. Contribución al estudio farmacológico de la Commelina pallida. I. Acción coagulante y vasoconstrictora. Gaceta Médica de México. 1944; 74: 140-145.

59. Oliva, I. et al. Neuropharmacological profile of hydroalcohol extract of Valeriana edulis ssp. procera roots in mice. Phytotherapy Research. 2004; 18 (4):290-296.

60. Baisch, A.L.; Urban, H.; Ruiz, A.N. Endothelium-dependent vasorelaxing activity of aqueous extracts of lyophilized seeds of Casimiroa edulis (AECe) on rat mesenteric arterial bed. Journal of Ethnopharmacology. 2004; 95 (2-3): 163-167.

61. Navarrete, A. et al. Gastroprotection and effect of the simultaneous administration of Cuachalalate (Amphipterygium adstringens) on the pharmacokinetics and anti-inflammatory activity of diclofenac in rats. The Journal of Pharmacy and Pharmacology. 2005; 57 (12): 1629-1636.

62. Delgado, G. et al. Antiinflammatory constituents from Heterotheca inuloides. Journal of Natural Products. 2001; 64 (7): 861-864.

63. Véase ก. 49. 
máximas y modalidades de presentación. El cuadro ${ }^{64}$ muestra la composición química de los extractos como se consignaba en la publicación y con el grado de precisión posible en la época.

El Cuadro 3 presenta otros productos de Codex resultantes de la combinación de diversos materiales y sustancias con los extractos ya referidos ${ }^{65}$. Se destaca en ellos la inclusión de medicamentos antiguos de uso popular, el predominio de purgantes y diuréticos, la presencia de indicaciones hoy en desuso, como el efecto antiescrofuloso y antiescorbútico del jarabe de rábano yodado, el cual sigue siendo requerido en la medicina doméstica de México aunque haya sido excluido de la farmacoterapia biomédica. Sin embargo, una parte significativa de los productos consignados en el Cuadro 3 , se encuentra hoy también al margen de la práctica médica doméstica.

\section{Conclusiones}

Los extractos fluidos de Codex son representativos de un momento determinado del proceso regulatorio de medicamentos en México: sus registros operaron solamente en el marco de la regulación que estuvo vigente entre 1928 y 1942. Ese hecho se asocia a la decreciente proporción de productos naturales en las farmacopeas mexicanas oficiales durante ese periodo: entre la edición de 1930 y la de 1952, las plantas contenidas pasan de 137 a 48, aún cuando habían alcanzado su máxima proporción en 1904, cuando llegaron a ser $609^{66}$.

Los reglamentos de 1928 y de 1942 definen básicamente las mismas figuras a registrar y certificar: se entendía por medicamentos «todos los productos naturales o sintéticos, simples o compuestos, destinados a un fin preventivo o curativo, tanto para el hombre como para los animales», de modo que esa categoría comprendía «medicinas de patente», «especialidades farmacéuticas» $\mathrm{y}$ «drogas», además de insecticidas, desinfectantes e incluso productos de tocador, higiénicos, de belleza y «demás similares». Las medicinas de patente eran definidas como «todo medicamento respecto del cual se haya obtenido derecho exclusivo conforme a la Ley de Patentes de Invención vigente», mientras que se entendía como especialidades «todo

\footnotetext{
64. Véase n. 49.

65. Véase n. 49

66. Hersch Martínez, n. 9, p. 390.
} 
medicamento, producto químico, droga natural o forma farmacéutica que lleve su designación científica o un nombre especial con el que se distinga y se ampare con una firma o marca». En todo caso, entre los cambios destacables entre un reglamento y otro se puede señalar que respecto a las «drogas simples» que no fuesen estudiadas o no figurasen en la Farmacopea o formularios legales, el Reglamento de 1928 exigía su clasificación botánica y el análisis de la planta, así como datos sobre sus propiedades farmacodinámicas y sobre sus aplicaciones terapéuticas; las disposiciones reglamentarias introducidas en 1942, en el caso de las «drogas vegetales», requerían, además de su clasificación botánica y el análisis de la planta, su lugar de procedencia, la parte empleada de la misma, datos sobre sus principios activos y su toxicidad ${ }^{67}$.

Para 1942 se abandona concepto de «droga simple» y la solicitud de definir «propiedades farmacodinámicas» y «aplicaciones terapéuticas» y se incorporan términos antes inexistentes en la regulación, como el del principio activo y el de toxicología como apartados de información requeridos en el caso de drogas vegetales, y en el caso de otros productos, la obligación de presentar datos sobre su acción farmacológica y sus dosis mínima y máxima según la edad, por cada vez que se administrase y por cada 24 horas. Hay otra incorporación significativa en el Reglamento de 1942, y es el requerimiento de que cualquier preparado que tuviese alguna novedad de índole terapéutica por su fórmula, dosis o vía de administración, aún cuando se tratase de una asociación de agentes medicinales ya conocidos, debería acompañarse de estudios clínicos-estadísticos realizados en establecimientos oficiales o bien en establecimientos particulares de «reconocida seriedad» con aval de médicos registrados ante el Departamento de Salubridad Pública ${ }^{68}$.

Los términos cambiaron, pero posiblemente menos que sus connotaciones; en todo caso, el contexto jugó su parte también: al requerimiento directo de validación experimental de eficacia por parte de las autoridades regulatorias, se añadía la necesidad de adecuación a un mercado y a una práctica donde esos productos contrastaban con el modelo de farmacoterapia de síntesis que se encontraba ya en plena consolidación ${ }^{69}$.

\footnotetext{
67. Departamento de Salubridad Pública, n. 5, art 1; n. 6, art. 1.

68. Departamento de Salubridad Pública, n. 5, art. 3; n. 6, arts. 13 y 14.

69. Hay que advertir que la cancelación de registro de medicamentos era motivada por una o varias causales diversas: porque los interesados no habían solicitado la revisión de los productos en
} 
La figura del extracto fluido estaba enmarcada en un ejercicio profesional médico y farmacéutico acorde con el avance técnico logrado en la época para el procesamiento de la materia médica, implicando un grado de estandarización suficiente para formular dosificaciones precisas hasta un determinado nivel cuantitativo, sin sacrificar con ello la adecuación de las composiciones finales al estado particular cambiante del enfermo. Esta posibilidad era determinante para el ejercicio de la formulación entendida como responsabilidad del terapeuta y preconizada desde su formación universitaria. Así, paradójicamente, el doctor Demetrio Mayoral Pardo, quien como director del Departamento de Salubridad Pública firmó la cancelación de gran parte de los registros de medicamentos a partir del reglamento de 1942, incluidos los extractos de Codex, en la misma época se ocupaba del arte de formular en su Manual de Terapéutica, destacando entre las «prescripciones medicamentosas» las preparaciones magistrales formuladas por los mismos médicos. La formulación, estandarizada y al margen del clínico, corresponde a una terapéutica estandarizada, distante de las representaciones ideales de individualización y diversidad detectables en la literatura médica ${ }^{70}$.

Mayoral Pardo plantea que la prescripción escrita - la receta- no concluye con la prescripción de la fórmula medicamentosa y con las indicaciones necesarias para el enfermo y para las personas encargadas de su cuidado, sino que comprende además los consejos o prescripciones de orden higiénico y dietético, que en numerosas circunstancias serán tan interesantes o más que las prescripciones medicamentosas; en esa perspectiva amplia, el autor advertía sobre la amplitud misma del término «régimen» y dividía a los agentes terapéuticos en aquellos de orden psíquico, de orden físico, de orden higiénico y dietético y los propiamente medicamentosos ${ }^{71}$.

Sin embargo, el médico, emplazado ante una modernidad que en pocos años se concretó en los efectos categóricos de la terapéutica de sustitución (antibióticos, esteroides, etc) acotará y estandarizará su prescripción en tipos determinados de enfermedades como procesos susceptibles de intervención

el plazo estipulado, o porque abandonaron la importación, elaboración y venta del producto, porque no habían cumplido en sí con los requisitos exigidos por el propio ordenamiento, o incluso por haber solicitado la cancelación los mismos interesados. Véase: Secretaría de Salubridad y Asistencia. Diario Oficial de la Federación. México. 7 Sept. 1944: 5.

70. Mayoral Pardo, Demetrio. Nociones de Terapéutica y Farmacodinamia [1939]. México: Talleres Gráficos de la Nación; 1946, p. 73-83.

71. Mayoral Pardo, n. 70, p. 74. 
sustitutiva. Nos encontramos en el inicio del proceso en el cual el medicamento deja de ser solamente un determinado compuesto para convertirse en el paradigma de la intervención curativa, en lo que se ha denominado el «imperialismo del objeto terapéutico» ${ }^{72}$. La declinación de los extractos fluidos como recursos terapéuticos se ubica en ese proceso y coincide con el acotamiento de la prescripción y la estandarización del «arte de formular» que, a su vez, atestiguan justamente el predominio de una lógica científica sobre una lógica del sujeto, las dos lógicas que de acuerdo con Lebrun atraviesan y organizan a la medicina contemporánea ${ }^{73}$.

El acotamiento del ejercicio prescriptivo - vinculado secundariamente con el abandono de los extractos fluidos susceptibles de combinación- sintetiza el proceso de transformación de la terapéutica, cuya estandarización pasa por la estandarización de la prescripción misma. Sin embargo, la mera existencia de esos productos en el mercado no implica que efectivamente se haya practicado de manera sistemática un «arte de formular» en el ejercicio médico anterior a la introducción de la farmacoterapia, aunque posiblemente las condiciones concretas de práctica cotidiana permitieron a algunos clínicos individualizar la formulación de remedios y brindar una prescripción diversificada en términos similares a los expresados por Mayoral Pardo.

¿Se debe retroceder y rescatar ese «arte de formular», en un planteamiento similar, por ejemplo, al del ámbito agropecuario con las prácticas de cultivo orgánico, o como proponía Schumacher respecto a la adecuación de la tecnología, o como plantean los teóricos del decrecimiento ante la evidencia de las graves consecuencias del hiperdesarrollo? ${ }^{74}$. En el caso de la viabilidad o pertinencia actual de los extractos fluidos, se puede aventurar un paralelismo con una reflexión que destaca Dubós de la arqueóloga Jacquetta Hawks, quien refería hace ya más de cincuenta años en relación al avance tecnológico existente a fines del siglo XVIII en Inglaterra:

«cuando recuerdo con tranquilidad la lenta posesión de la Gran Bretaña por sus pobladores, no puedo resistir a la conclusión de que la relación adquirió la máxima intimidad, el temple más sensible hace cosa de doscientos años. A mediados del Siglo XVIII los hombres habían triunfado, la tierra era suya, pero

72. Lebrun, Jean-Pierre. De la maladie médicale. Bruselas: Ed. De Boeck Université; 1993, p. 54-55.

73. Lebrun, n. 72, p. 43.

74. Schumacher, Ernest F. Lo pequeño es hermoso. Madrid: H. Blume Ediciones; 1978; Latouche, Serge. El planeta de los náufragos. Ensayo sobre el posdesarrollo. Madrid: Acento Editorial; 1993. 
aún no la habían sometido y ultrajado. El reino silvestre había sido restringido a las montañas, y allí por primera vez podía admirársele sin peligro. Las comunicaciones eran suficientes para ligar el país en una unidad inexistente desde que era una provincia romana, pero no eran todavía tan fáciles que destruyeran el carácter local y la natural libertad del individuo que el estar lejos le concede en abundancia. Los ricos y los pobres sabían cómo usar el país para levantar bellos edificios y para agruparlos con gracia instintiva. Por haber crecido juntos, para servirse mutuamente, la ciudad y el campo disfrutaron entonces de un momento de equilibrio». ${ }^{75}$

Así, aún sin proyectar una imagen idílica de la relación entre clases sociales, sin añorar precariedades cuya distribución diferencial persiste en todo el mundo y sin pretender pasar por alto los indiscutibles logros del desarrollo científico y tecnológico en el campo de la farmacoterapia y la farmacia, hubo un momento en que un determinado estadio del desarrollo tecnológico - en éste caso en el campo de la farmacia- permitiría optimizar el uso terapéutico de los recursos naturales sin llegar al punto de avasallar la libertad y la versatilidad prescriptiva de los clínicos.

En ese sentido, en la producción analizada en este trabajo conviven lenguajes diversos: a) el de un saber técnico que facilita la disponibilidad de la materia médica sin cerrar el paso a cierto margen de libertad prescriptiva del médico, basada en la combinación de extractos de diversa indicación ante un paciente en quien coinciden diversas expresiones de enfermedad; b) el de un conjunto de padecimientos donde la serie de indicaciones de uso de los productos comerciales, como aproximado espejo de la realidad, corresponde a un conjunto de síntomas y signos que motiva la consulta; c) el de una terminología que refleja una precisión relativa en las propiedades sintomáticas o terapéuticas de los extractos, reconociendo la relevancia de las demandas de respuesta inmediata por parte de los pacientes hacia el facultativo, aún cuando, como es frecuente, el origen del padecimiento no necesariamente se precise en la primera visita.

Ahora bien, ¿Cuáles de esas necesidades y requerimientos de profesionales y legos persisten hoy? ¿Acaso no persiste la necesidad de dos ejes antes insistentemente imbuidos en la formación médica, el de la individualización diagnóstica y el de la diversidad terapéutica, presentes de manera 
indirecta en la producción analizada? ${ }^{76}$ ¿Podría la técnica farmacéutica hoy coadyuvar en el rescate de la adecuación prescriptiva, en lugar de ser funcional a la estandarización de los pacientes por la vía de tratamientos idénticos que pasan por alto la heterogeneidad de los individuos y su dimensión fisiopatológica particular y personal?

A su vez, al margen de una detenida revisión pertinente, muchos de los efectos atribuidos a las especies que daban origen a los extractos fluidos de Codex - propiedades avaladas oficialmente en México por el entonces denominado Departamento de Salubridad Pública- pueden considerarse fundamentadas a partir de estudios experimentales disponibles hoy. La paradoja es que más de medio siglo después, esos mismos productos, elaborados actualmente por la empresa que se originó en Codex, carecen de registro como medicamentos, a pesar de que hoy se dispone de mayor y más precisa información experimental al respecto.

Elemento complementario en esa paradoja, es que respecto a una proporción considerable de las atribuciones de los extractos entonces registrados, tampoco se han generado estudios experimentales que invaliden expresamente esas indicaciones, las cuales fueron definidas por reconocidos académicos que se apoyaron en información procedente de la tradición popular, de la tradición académica, del conocimiento médico empírico y de estudios experimentales de la época.

Así, información como la analizada constituye un punto de partida pertinente en la necesaria revisión del conjunto de atribuciones surgidas de una experiencia que ha sido insuficientemente ponderada hoy. En este sentido, la confrontación de las indicaciones de esos extractos con la información experimental obtenida años después respecto a las mismas especies utilizadas en su confección, exige el cotejo de términos que no son equivalentes, y que expresan una aproximación cualitativa y clínica legítima pero soslayada, ante un modelo experimental que focaliza efectos precisos a nivel molecular incidiendo en receptores específicos ${ }^{77}$. A su vez, una tarea complementaria para ese fin es también el análisis de una terminología que ha cambiado, expresando dos momentos diferentes en la construcción social de la realidad terapéutica. De ahí que resulte pertinente la revisión pormenorizada de las indicaciones, usos y efectos, ligando las 
atribuciones de entonces con los datos actuales; si muchas especies incluidas en los productos Codex hoy cuentan con información experimental sólida, sus extractos deben ser sometidos a ensayos clínicos. Sin embargo, no se llevan a cabo, dado el carácter determinante de esos ensayos en el proceso de conformación de una determinada sustancia en una mercancía farmacéutica ${ }^{78}$.

Ahora bien, en la relación de especies de uso etnobotánico de la Extrafarmacopea anexa a la Farmacopea Herbolaria editada en México por la Secretaría de Salud en $2001^{79}$, figuran muchas de las plantas utilizadas en los extractos fluidos de Codex, y en la Ley General de Salud actualmente vigente se han abierto espacios para la regulación de este tipo de productos; los medicamentos herbolarios y los remedios herbolarios son definidos respectivamente en ese marco como:

«los productos elaborados con material vegetal o algún derivado de éste, cuyo ingrediente principal es la parte aérea o subterránea de una planta o extractos y tinturas, así como jugos, resinas, aceites grasos y esenciales, presentados en forma farmacéutica, cuya eficacia terapéutica y seguridad ha sido confirmada científicamente en la literatura nacional o internacional» ${ }^{80}$.

« (...)preparado de plantas medicinales, o sus partes, individuales y combinadas y sus derivados, presentado en forma farmacéutica, al cual se le atribuye por conocimiento popular o tradicional, el alivio para algunos síntomas participantes o aislados de una enfermedad. Los remedios herbolarios no contendrán en su formulación sustancias estupefacientes o psicotrópicas, ni ningún otro tipo de fármaco alopático u otras sustancias que generen actividad hormonal, antihormonal o cualquier otra sustancia en concentraciones que represente riesgo para la salud» ${ }^{81}$.

Estas figuras regulatorias contribuyen desde 1996 a dar una salida al reto de la regulación de los extractos vegetales, pero sigue siendo casi nulo el impulso de instancias oficiales a su investigación clínica, determinante

78. Hersch Martínez, n. 10, p. 170-178. Pignarre, n. 13: «se cuentan con los dedos de una mano los estudios clínicos no ordenados y formateados por la industria farmacéutica, que ha dedicado una parte considerable de sus recursos a este episodio clave en la puesta a punto de todo medicamento» (p. 81).

79. Secretaría de Salud. Farmacopea Herbolaria de los Estados Unidos Mexicanos. México: Comisión Permanente de la Farmacopea de los Estados Unidos Mexicanos; 2001.

80. Secretaría de Salud. Ley General de Salud, Art. 224. México; 1998.

81. Secretaría de Salud. Reglamento de Insumos para la Salud, Art. 88. México: 1998. 
para el registro de los medicamentos herbolarios, al tiempo que las mismas autoridades declaran como insuficientemente definido el término de «conocimiento popular o tradicional», cuya fundamentación constituye el aval de los remedios herbolarios ${ }^{82}$.

Estas figuras han sido utilizadas en particular por la industria farmacéutica extranjera para lanzar al mercado una «nueva» modalidad de producto, el «fitomedicamento», que si bien enriquece la gama de recursos terapéuticos al aportar el potencial sinérgico de extractos vegetales totales, ya que no se reduce a principios moleculares aislados, no implica en sí precios más accesibles, ni la posibilidad de individualizar formulaciones para el clínico, ni el aprovechamiento de la diversidad florística medicinal del país; de hecho, a diez años de la introducción de la figura del medicamento herbolario, la mayor parte de los registros otorgados corresponden, paradójicamente, a productos importados, elaborados con especies medicinales que no existen en México.

Estos elementos remiten justamente al contexto del proceso regulatorio, el cual ha cambiado al supeditarse progresivamente la práctica validatoria de los recursos terapéuticos potenciales a dos pautas dominantes: a) un paradigma biomédico que no sólo sigue descalificando el saber popular a pesar de la introducción de las categorías regulatorias del medicamento y del remedio herbolarios, sino que excluye los conocimientos empírico-médicos procedentes de la propia práctica biomédica clínica; y b) la carencia de una perspectiva nacional para impulsar el aprovechamiento y desarrollo de los propios saberes, recursos y posibilidades terapéuticas existentes en el país.

En ambas pautas, hay textos de referencia terapéutica que atestiguan en el marco de la medicina oficial de fines del siglo XIX y principios del XX en México la existencia previa de una perspectiva contraria. En el caso del saber proveniente de la experiencia personal de los clínicos, tenemos el Formulario de la Facultad Médica Mexicana, publicación conformada por los testimonios de la práctica terapéutica de diversos médicos de todo el país ${ }^{83}$. En el caso de una perspectiva nacional de los recursos terapéuticos,

82. Hersch Martínez, n. 10, p. 444-449. Enríquez Rubio, Ernesto; Frati Munari, Alberto; González Pier, Eduardo, comps. Hacia una política farmacéutica integral para México. México: Comisión Federal para la Protección contra Riesgos Sanitarios; 2005, p. 49-55.

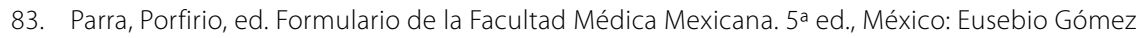
de la Puente; 1921. 
se encuentra la Farmacología Nacional producida por el Instituto Médico Nacional en plena época de la Revolución ${ }^{84}$. Ambas pautas refieren a la optimización de las posibilidades inherentes a los saberes y recursos de un país muy diverso en términos de naturaleza y cultura como México.

Todo ello lleva a destacar que en la validación del potencial terapéutico de esos saberes y recursos, se requiere hoy un proyecto que detecte la diversidad de sus expresiones, armonizando una rigurosa investigación experimental con las pautas que emergen de diversas fuentes de conocimiento, incluyendo las históricas. Los recursos que alguna vez fueron reconocidos como eficaces y seguros y que en mayor o menor grado siguen gozando de dicho reconocimiento en amplios sectores de la población, - como es el caso de la mayor parte de los extractos fluidos referidos en este trabajo- se encuentran a la espera de métodos de validación fincados en una evaluación que integre las diversas expresiones de su potencial. En ese sentido, la historia social de la terapéutica tiene implicaciones operativas pertinentes ante problemas actuales, relacionados con la calidad de la atención médica y con la regulación y disponibilidad de medicamentos. Parafraseando al ya referido Schumacher al aludir a la economía, ¿cómo sería la terepéutica biomédica si la gente importara?

84. Instituto Médico Nacional. Farmacología Nacional. México: Tipografía Económica; 1913. 